
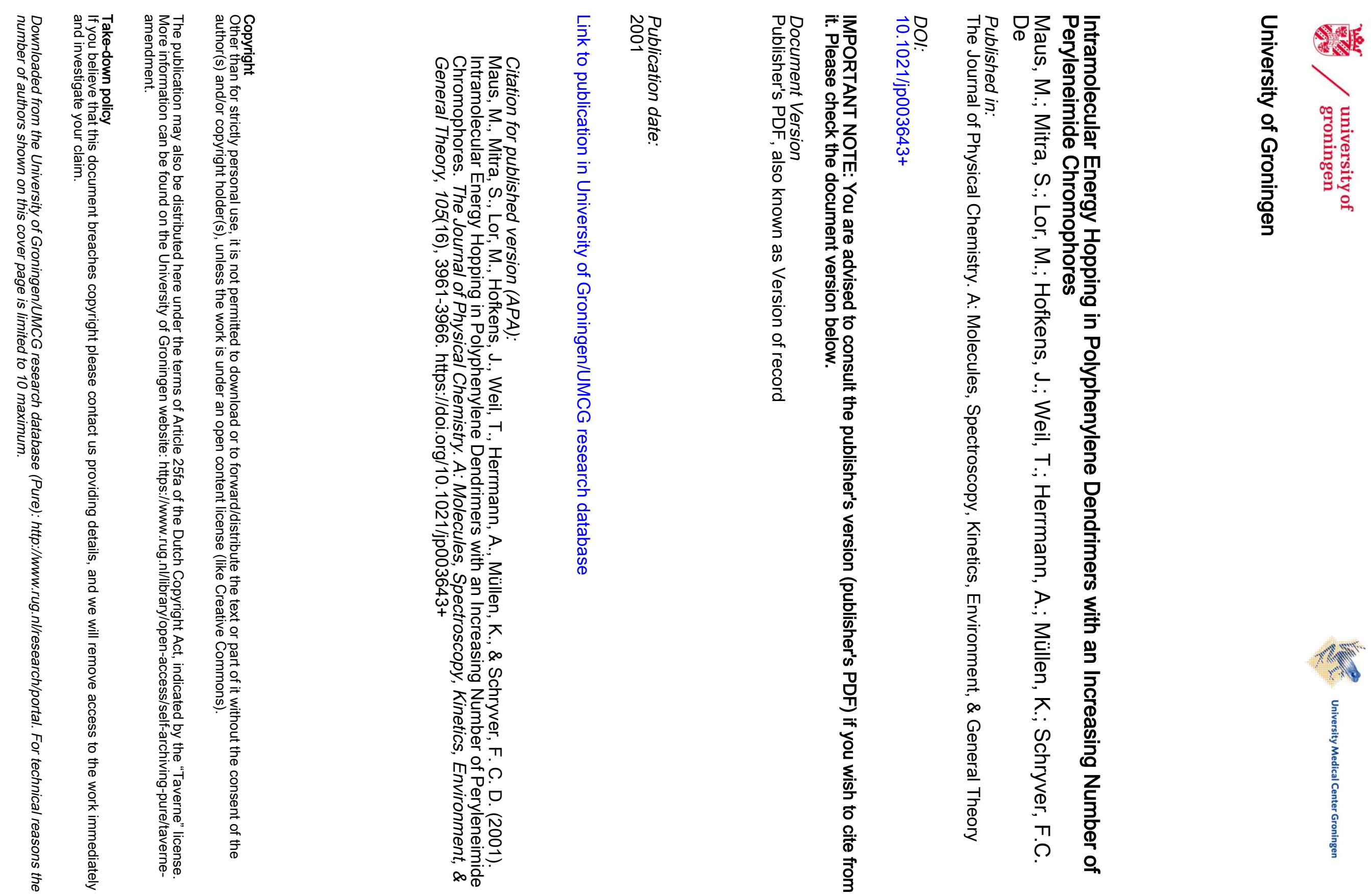


\title{
Intramolecular Energy Hopping in Polyphenylene Dendrimers with an Increasing Number of Peryleneimide Chromophores
}

\author{
M. Maus, S. Mitra, M. Lor, J. Hofkens, T. Weil, ${ }^{\dagger}$ A. Herrmann, ${ }^{\dagger}$ K. Muillen, ${ }^{\dagger}$ and \\ F. C. De Schryver* \\ Department of Chemistry, Katholieke Universiteit Leuven, Celestijnenlaan 200F, 3001 Heverlee, Belgium \\ Received: October 4, 2000; In Final Form: January 24, 2001
}

\begin{abstract}
The photophysical properties and the excitation energy transfer (EET) process of a series of first generation dendrimers having a rigid tetrahedral core and different numbers of peryleneimide chromophores at the rim were investigated by steady-state and time-resolved fluorescence spectroscopy in toluene. It was shown that the model dendrimer containing only one chromophore decays monoexponentially with a time constant of $4.2 \mathrm{~ns}$. In the multichromophoric compounds an additional long decay time of $7.4 \mathrm{~ns}$ assigned to an "excimerlike" state was observed, and its contribution increases with the number of chromophores. The time-resolved polarization measurements resulted in a nanosecond relaxation component due to the rotation of the entire dendritic structure. However, for the compounds having more than one chromophore, an additional fast anisotropy decay time of 100-200 ps was observed which corresponds to Förster type EET among the chromophores.
\end{abstract}

\section{Introduction}

Recently much attention has been devoted to the controlled synthesis and characterization of three-dimensional molecular systems known as dendrimers since their highly branched regular structures can be used as building blocks for the fabrication of target designed molecules. ${ }^{1,2}$ The three-dimensional shape of the dendrimers is determined by the interior building block (core) and the peripheral groups. In contrast to the other macromolecules, the size, shape, and functionality of the dendrimers can be defined at the molecular level. Application of the dendrimers have been suggested in several fields such as guest-host chemistry, ${ }^{3}$ analytical chemistry, ${ }^{2}$ optoelectronics, ${ }^{4}$ catalysis, ${ }^{5}$ biology, ${ }^{6}$ and medicine. ${ }^{7}$ Dendrimers are also used to mimic the photophysical processes of the basic photosynthetic light-harvesting antennae systems. ${ }^{8-12}$

Synthetic procedures ${ }^{13}$ and physical organic studies ${ }^{14-17}$ on dendrimer molecules with a polyphenylene core decorated with different numbers of peryleneimide chromophores on the surface have been reported. The combination of picosecond single photon timing (SPT) and femtosecond transient absorption, ${ }^{14}$ femtosecond fluorescence upconversion, ${ }^{15,16}$ single-molecule spectroscopy, ${ }^{12,17}$ and atomic force microscopy ${ }^{18}$ lead to an understanding of the time- and space-resolved behavior of these dendrimers. Particularly for the time-resolved properties of the peryleneimide dendrimers with a biphenyl core, ${ }^{14-16}$ it was shown that although the excited state decays mainly with the fluorescence decay time of about $4 \mathrm{~ns}$ for the single chromophoric system along with a $7.5 \mathrm{~ns}$ component for the

\footnotetext{
* Corresponding author. E-mail: Frans.DeSchryver@ Chem.KULeuven.ac.be.

$\dagger$ Present address: Max-Planck-Institut für Polymerforschung, Ackermannweg 10, 55128 Mainz, Germany.
}

"dimerlike" state resulting from chromophore-chromophore interaction for the dendrimers having multiple chromophores, a number of other kinetic components are present on the ultrashort time scale.

However, the studies mentioned above are for dendrimers involving peryleneimide chromophores and polyphenylene arms but with a biphenyl core leading to a three-dimensional structure with a biconical shape. ${ }^{12}$ It was shown that due to the conformational dynamics the peryleneimide chromophores are distributed unevenly in different environments at the rim of the dendrimer. ${ }^{12}$

To get a better control over the spatial distribution of chromophores and a better insight into the interaction in the excited state, it is desirable to have them distributed on a more spherical surface. Recently, this goal has been achieved with the synthesis of a series of first generation peryleneimide dendrimers with a rigid tetrahedral central core. ${ }^{19}$ The number of peryleneimide chromophores (PI, Chart 1) is varied from 1 to $4(\mathbf{G 1 R} x ; x=1-4, \mathbf{I}-\mathbf{I V}$, Chart 1$)$ along the branches of the dendrimer in the direction of the corners of a tetrahedron due to the central $\mathrm{sp}^{3}$ carbon of the core. In this way, it is possible to get a more controlled orientation of the polyphenylene building blocks around the central core. As a result, the possibility of energy transfer in multichromophoric systems can be studied more systematically.

In this contribution, we report the solution phase photophysics of these dendrimers studied by steady-state and picosecond timeresolved spectroscopic techniques.

\section{Experimental Section}

Materials. The synthesis of these rigid dendrimers having a tetraheral core decorated with a different number of perylene-

\subsection{1/jp003643+ CCC: $\$ 20.00 \quad$ C 2001 American Chemical Society}


CHART 1: Structures of the Molecules Used in This Study
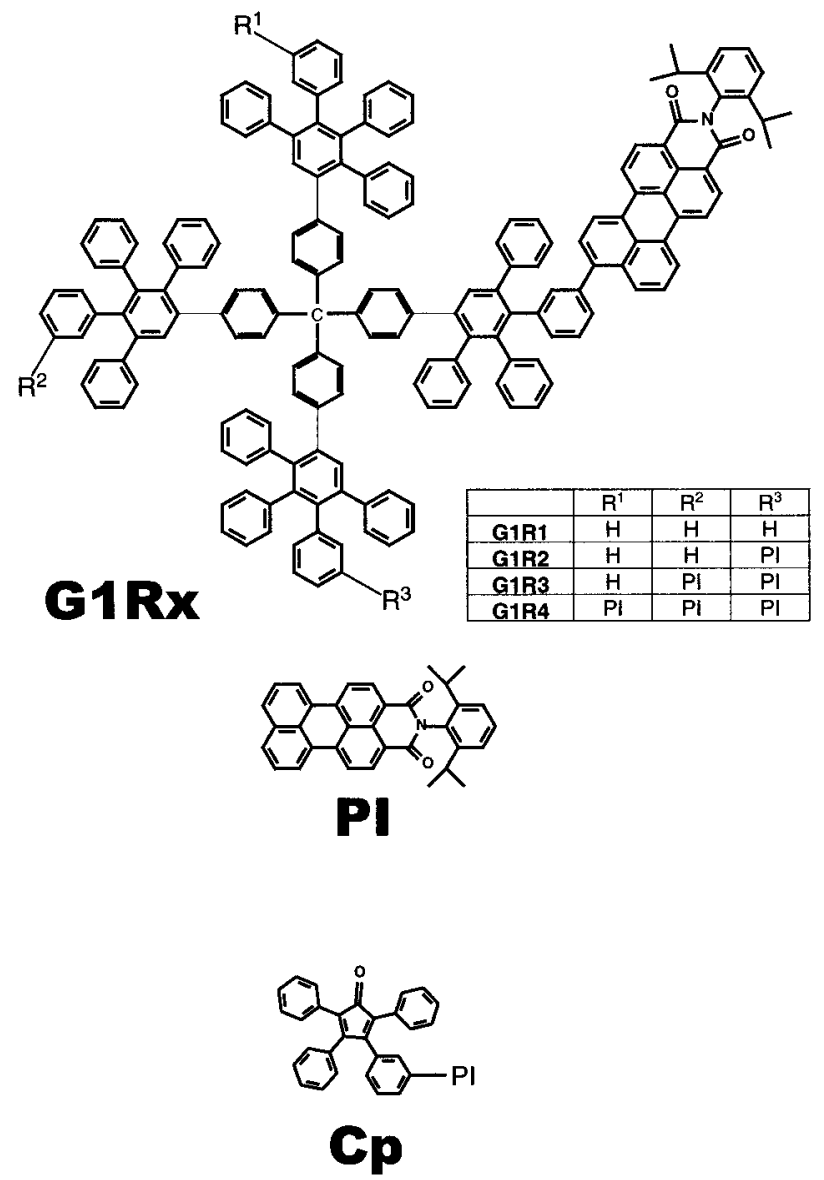

imide chromophores on the polyphenylene rim utilizes the tetraphenylcyclopentadienone (CP, Chart 1) which carries the peryleneimide chromophore. While the symmetric G1R4 is made via Diels-Alder cycloaddition of $\mathbf{C P}$ to a core molecule with four ethinyl functions, the "desymmetrized" species G1R1, G1R2, G1R3 require a novel approach which is being published separately. ${ }^{19}$

The samples were dissolved in toluene (Aldrich) to have an optical density just below 0.1 in a $1 \mathrm{~cm}$ cell at the absorption maximum $(490 \mathrm{~nm})$ which corresponds to a concentration of $\sim 10^{-7} \mathrm{M}$.

Steady-State Measurements. Steady-state absorption and corrected fluorescence spectra were recorded with Lambda 40 (Perkin-Elmer) and SPEX spectrophotometers, respectively. The fluorescence quantum yields have been determined using Rhodamine 101 and Cresyl violet perchlorate as a reference. ${ }^{20}$

Picosecond Time-Resolved Measurements. The fluorescence decay times have been determined by the single photon timing method using a laser and detection setup described previously. ${ }^{14,21}$ In brief, the second harmonic of a Ti:sapphire laser (Tsunami, Spectra Physics) has been used to excite the samples at $488 \mathrm{~nm}$ with a repetition rate of $4.09 \mathrm{MHz} .{ }^{14}$ The detection system ${ }^{21}$ consists of a subtractive double monochromator (9030DS, Sciencetech) and a microchannel plate photomultiplier (R3809U, Hamamatsu). A time-correlated single photon counting PC module (SPC 630, Picoquant $\mathrm{GmbH}$ ) which has the two constant fraction discriminators (CFD), a time-toamplitude converter (TAC), and an analog-to-digital converter (ADC) on board was used to obtain the fluorescence decay histograms in 4096 channels with time increments of 5 or 10 ps. The fluorescence decays have been recorded at three different orientations of the emission polarizer relative to the polarization plane of the excitation light, i.e., $54.7^{\circ}$ (magic angle), $0^{\circ}\left(I_{\mathrm{par}}\right)$, and $90^{\circ}\left(I_{\text {perp }}\right)$. The magic angle decays were analyzed globally with a time-resolved fluorescence analysis (TRFA) software. ${ }^{22}$ The quality of the fits has been judged by the fitting parameters such as $\chi^{2}(<1.2)$ and $Z \chi^{2}(<3)$ as well as by the visual inspection of the residuals and autocorrelation function. ${ }^{23}$ The anisotropy decay as calculated from eq 1 were fitted by a linear combination of exponentially decaying functions using the Levenberg-Marquard $\chi^{2}$ minimization on the basis of eq 2, where $N$ is the number of data points and $P$ is the number of free parameters in the fit function. The anisotropy decay analysis was performed with the commercial ORIGIN 6.0 (Microcal Software Inc.) program, and the results were double-checked with the TRFA program ${ }^{22}$ which also takes pulse deconvolution into account.

$$
\begin{array}{r}
r(t)=\frac{I_{\text {par }}(t)-I_{\text {perp }}(t)}{I_{\text {par }}(t)+2 I_{\text {perp }}(t)} \\
\chi^{2}=\frac{\sum_{i=1}^{N}\left[y_{i}-f\left(x_{i}\right)\right]^{2}}{N-P}
\end{array}
$$

\section{Theoretical Model for Energy Hopping}

Our theoretical modeling of the observed dynamics involves excitation energy hopping among $i=2,3$, or 4 identical chromophores, which, in the simplest approach possible, are perfectly arranged with in average equal distances and orientations toward each other. This allows to consider a single rate constant $k_{\text {eet }}$ of energy transfer. From this assumption it follows that dynamics of incoherent energy hopping are described by the master equation

$$
\frac{\mathrm{d} \mathbf{P}}{\mathrm{d} t}=\mathbf{K} \mathbf{P}
$$

where $\mathbf{P}$ is the vector of the time-dependent individual excitation probabilities

$$
\mathbf{P}(t)=\left(\begin{array}{c}
P_{1}(t) \\
\cdots \\
P_{i}(t)
\end{array}\right)
$$

and $\mathbf{K}$ is the matrix of rate constants

$$
\mathbf{K}=\left(\begin{array}{ccc}
-(i-1) k_{\mathrm{eet}}-\tau_{\mathrm{f}}^{-1} & \cdots & k_{\mathrm{eet}} \\
\cdots & \cdots & \cdots \\
k_{\mathrm{eet}} & \cdots & -(i-1) k_{\mathrm{eet}}-\tau_{\mathrm{f}}^{-1}
\end{array}\right)
$$

where all off-diagonal elements are equal to $k_{\text {eet }}$ and the diagonal elements are dependent on $k_{\text {eet }}$, the number of chromophores $i$, and the fluorescence lifetime $\tau_{\mathrm{f}}$ of the chromophores. The fluorescence intensity decay $I_{\mathrm{f}}(t)$ can hence be described by a single-exponential function with arbitrary amplitude $\alpha$ according to

$$
I_{\mathrm{f}}(t)=\sum_{1}^{i} \underset{i}{-\alpha} P_{i}(t)=\alpha \mathrm{e}^{-t / \tau_{\mathrm{f}}}
$$

which does therefore not allow one to determine $k_{\text {eet }}$. 


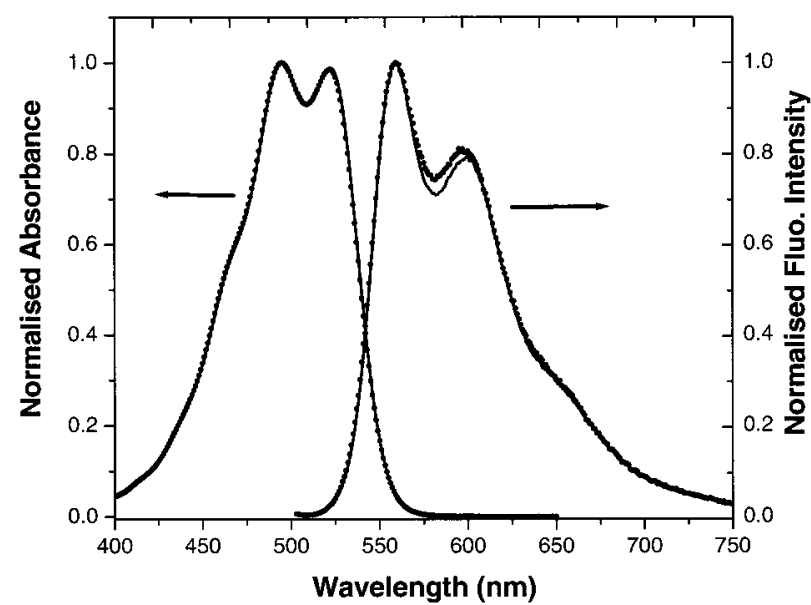

Figure 1. Steady-state absorption and emission spectra of the model compound G1R1 (solid line) and G1R4 (dots).

The observable quantity that reflects the energy hopping dynamics is the time-resolved fluorescence anisotropy $r(t){ }^{8,24-29}$ Since the anisotropy contribution from excited chromophores populated by energy transfer for an energy-hopping system can be assumed to be zero with an error of less than $2 \%$ for random chromophore orientation, ${ }^{24,25}$ the ordinary differential equation system (eq 7) for the time-dependent anisotropy residing on individual chromophores, where the rate constants for depolarization are $k_{\text {eet }}$ and the inverse of the anisotropy relaxation time $\Theta_{\text {rot }}^{-1}$ for the rotation of the whole molecule, needs to be solved only for the anisotropy $r_{1}$ of the initially exited chromophore ${ }^{8,26-28}$ with the initial conditions that $r_{1}(0)$ equals the limiting anisotropy $r_{0}$ and that $r_{j \neq 1}=0$ yielding eq 8 .

$$
\begin{aligned}
& \frac{\mathrm{d}}{\mathrm{d} t}=\left(\begin{array}{c}
r_{1}(t) \\
\cdots \\
r_{i}(t)
\end{array}\right)= \\
& \left(\begin{array}{ccc}
-(i-1) k_{\mathrm{eet}}-\Theta_{r o t}^{-1} & \cdots & k_{\mathrm{eet}} \\
\cdots & \cdots & \cdots \\
k_{\mathrm{eet}} & \cdots & -(i-1) k_{\mathrm{eet}}-\Theta_{\mathrm{rot}}-1
\end{array}\right)
\end{aligned}
$$

This simple model thus predicts a double-exponential anisotropy

$$
r(t)=r_{1}(t)=\frac{r_{0}}{i}\left[\mathrm{e}^{-t / \Theta \mathrm{rot}}+(i-1) \mathrm{e}^{-\left(i k e \mathrm{e} t+\Theta_{\mathrm{rot}}{ }^{-1}\right) \mathrm{t}}\right]
$$

decay with an amplitude ratio of $(i-1)$. In case of absent rotational movement $\left(\Theta_{\text {rot }}{ }^{-1}=0\right)$ a constant term $r_{0} / i$ gives the well-known leveling-off at long times. ${ }^{8,26-29}$

\section{Results}

Steady-State Spectra. The steady-state absorption and fluorescence spectra of all the dendrimers $\mathbf{I}-\mathbf{I V}$ which contain only the peryleneimide chromophore are very similar. Representative examples for the model compound with one chromophore, G1R1, and with maximum number of four chromophores, G1R4, in toluene are shown in Figure 1. The absorption spectra of all the dendritic compounds in toluene give two vibronic maxima at 495 and $520 \mathrm{~nm}$. The fluorescence spectra shows maxima at 560 and $595 \mathrm{~nm}$. The spectral properties and the high fluorescence quantum yields $\left(\phi_{\mathrm{f}}=0.95\right.$ $\pm 0.05)$ of all dendrimers $\mathbf{I}-\mathbf{I V}$ are practically the same within error. Only the fluorescence full width at half-maxima (fwhm) increases slightly from $2680 \mathrm{~cm}^{-1}$ for G1R1 to $2750 \mathrm{~cm}^{-1}$ for G1R4 with the number of chromophores.

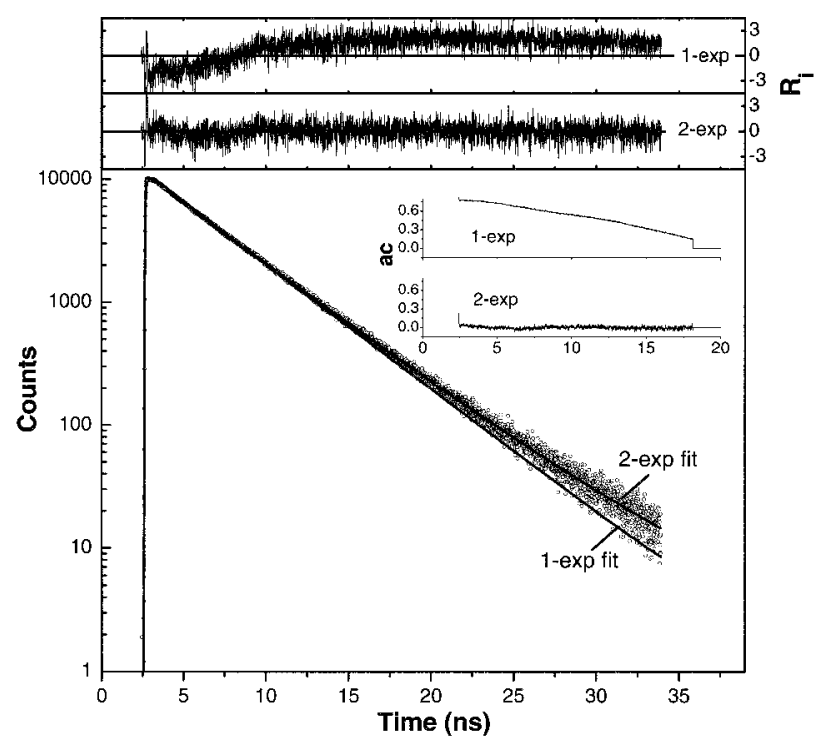

Figure 2. Time-resolved fluorescence decay of G1R4 with mono- and biexponential fitting. The upper panel shows the distribution of weighted residuals $\left(R_{i}\right)$, and in inset the autocorrelation (ac) functions are given in both cases.

TABLE 1: Fluorescence Decay Times $\left(\boldsymbol{\tau}_{i}\right)$ and Associated Relative Amplitudes $\left(\alpha_{i}\right)$ for G1R1-G1R4 Measured in Toluene at Room Temperature Using $\lambda_{\text {exc }}=488 \mathbf{n m}^{a}$

\begin{tabular}{lccccccc}
\hline & & & \multicolumn{2}{c}{$\lambda_{\text {flu }}=600 \mathrm{~nm}$} & & \multicolumn{2}{c}{$\lambda_{\text {flu }}=725 \mathrm{~nm}$} \\
\cline { 4 - 5 } & $\tau_{1}(\mathrm{~ns})$ & $\tau_{2}(\mathrm{~ns})$ & $\alpha_{1}(\%)$ & $\alpha_{2}(\%)$ & & $\alpha_{1}(\%)$ & $\alpha_{2}(\%)$ \\
\hline I & 4.25 & & 100 & 0 & & \\
II & 4.25 & $7.4^{b}$ & 99.2 & 0.8 & & 98.7 & 1.3 \\
III & 4.25 & $7.4^{b}$ & 98.1 & 1.9 & & 96.2 & 3.8 \\
IV & 4.25 & 7.4 & 96.0 & 4.0 & & 93.7 & 6.3
\end{tabular}

${ }^{a}$ The error of $\tau_{1}$ is $\pm 0.05 \mathrm{~ns}$ and that of $\tau_{1}$ is $\pm 0.6 \mathrm{~ns}$. ${ }^{a}$ Kept fixed for II and III as obtained from IV (see text for details).

Time-Resolved Fluorescence Measurements. To examine the properties of the fluorescent states for the dendrimers more closely, fluorescence decay times for all the dendrimers were determined in toluene by single-photon-timing detecting the emission under magic angle condition. A representative decay trace for G1R4 is shown in Figure 2, and the respective decay parameters are collected in Table 1 . The fluorescence intensity of the dendrimer having only one chromophore (G1R1) decays single exponentially with a decay time of $4.25 \pm 0.05 \mathrm{~ns}$. However, as the number of chromophores is increased in the dendrimer, a small contribution of an additional long decay component of $7.4 \pm 0.6 \mathrm{~ns}$ is found essential to fit the experimental data as demonstrated by the distribution of weighted residuals $\left(R_{i}\right)$ and autocorrelation function $(\mathrm{ac})$ for a mono- and biexponential fit in Figure 2. It has to be noted that the amplitude of this long decay component is however very small in G1R2 and G1R3. Thus, to minimize the error in the fit procedure, the decay time of 7.4 ns obtained from G1R4 was kept fixed in the analysis of G1R2 and G1R3 to compare better the corresponding amplitudes In addition, the relative amplitude of the longer decay time is larger at the red edge of the fluorescence spectrum for all multichromophoric dendrimers as reported in Table 1 by the comparison of results obtained at 600 and $725 \mathrm{~nm}$ emission.

Time-Resolved Fluorescence Polarization Measurements. From time-resolved fluorescence polarization measurements, the anisotropy decay times $\left(\Theta_{j}\right)$ and the associated anisotropy $\left(\beta_{j}\right)$ have been determined for all the compounds using eq 9.

For the dendrimer having only one chromophore (G1R1), a 
TABLE 2: Fitting Parameters of the Fluorescence Anisotropy Decays Measured for G1R1-G1R4 in Toluene with $\lambda_{\text {exc }}=488$ and $\lambda_{\text {flu }}=600 \mathrm{~nm}$ and Calculated Rotor Diameters $\left(d_{\text {rot }}\right)$ and Average Chromophore-Chromophore Distances $\left(d_{\text {FRET }}\right)$

\begin{tabular}{lcccccccc}
\hline compd & $r_{0}$ & $\begin{array}{c}\Theta_{1} \\
(\mathrm{~ns})\end{array}$ & $\begin{array}{c}\Theta_{2} \\
(\mathrm{~ns})\end{array}$ & $\beta_{1}$ & $\beta_{2}$ & $\begin{array}{r}\beta_{2} / r_{0} \\
(\%)\end{array}$ & $\begin{array}{c}d_{\text {rot }} \\
(\AA)^{a}\end{array}$ & $\begin{array}{c}d_{\text {FRET }} \\
(\AA)^{b}\end{array}$ \\
\hline I & 0.38 & 0.95 & & 0.38 & & 0 & 23.3 & \\
II & 0.31 & 1.1 & 0.20 & 0.16 & 0.15 & 48 & 24.3 & 26.5 \\
III & 0.28 & 1.2 & 0.13 & 0.10 & 0.18 & 63 & 25.3 & 26.1 \\
IV & 0.24 & 1.3 & 0.11 & 0.08 & 0.16 & 66 & 25.9 & 26.5
\end{tabular}

${ }^{a}$ Calculated from eqs 10 and $14 .{ }^{b}$ Calculated from eqs 14 and 15 using $i$ equal to 2,3 , and 4 for II-IV, respectively. See text for details.

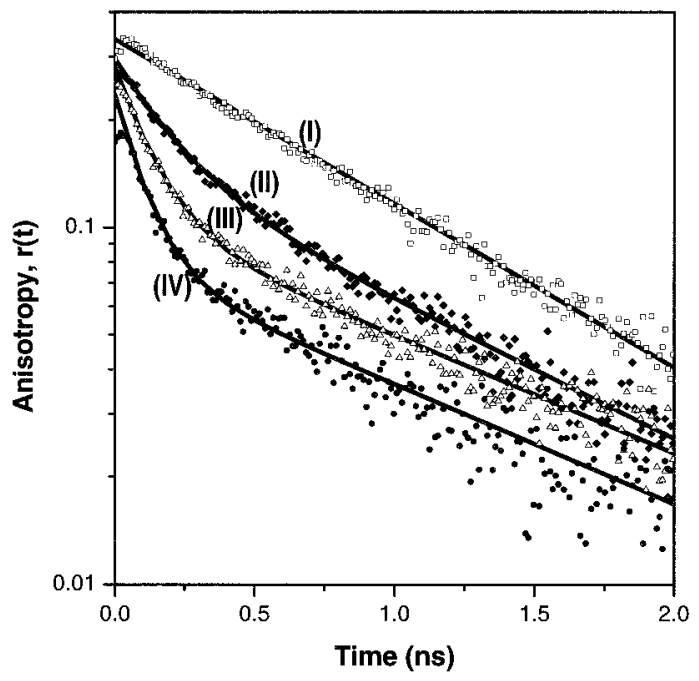

Figure 3. Time-resolved fluorescence anisotropy decays (scattered symbol) and fits (solid line) for the dendrimers G1R1 (I), G1R2 (II), G1R3 (III), and G1R4 (IV) in toluene. The excitation and emission wavelengths are 488 and $600 \mathrm{~nm}$, respectively.

monoexponential fit of the anisotropy decay function is sufficient which gives a relaxation time of $\Theta_{1}=950 \pm 30$ ps with $\beta_{1}=$ $r_{0}=0.38$ (Table 2). However, the anisotropy decay functions for the dendrimers having more than one chromophore (G1R2G1R4) can only be fitted with two exponential decay functions (Figure 3). The long depolarization time constant is similar to that obtained for G1R1. The magnitude of this long time component increases with the number of chromophores from $1.1 \pm 0.04 \mathrm{~ns}$ for G1R2 to $1.3 \pm 0.07$ for G1R4 while the value of the fast component $\left(\Theta_{2}\right)$ changes from $200 \pm 30$ ps for G1R2 to $110 \pm 20$ ps in G1R4 (Table 2).

$$
r(t)=\sum \beta_{j} \exp \left(-t / \Theta_{j}\right) \quad r_{0}=\sum_{j} \beta_{j}
$$

\section{Discussion}

Fluorescence from a Localized and Excimer-like Species. The steady-state properties of all the dendrimers $(\mathbf{I}-\mathbf{I V})$ are practically the same (Figure 1) with the exception of some broadening of the fluorescence spectra with increasing number of chromophores. This result is different from the observations made for the higher generation peryleneimide dendrimers having a biphenyl flexible core and multiple chromophores as reported elsewhere, ${ }^{14}$ where a number of properties, such as spectral shape, fluorescence maxima, and fluorescence quantum yield are influenced by the formation of an excimer-like species. From the small difference in the spectral width, the assumption of an excited-state excimer-like chromophore-chromophore interaction is possible but not conclusive.
Better insight into the extent of excimer-like emission is obtained from the fluorescence decays where only for the multichromophoric dendrimers a long decay component of 7.4 ns is observed along with the typical peryleneimide fluorescence decay time of $4.25 \mathrm{~ns}$ as obtained for the monochromophoric model compound G1R1. The attribution of this long time constant can be made to an "excimer-like" species as the decay time is similar to that reported for the higher generation dendrimers having a flexible biphenyl core. ${ }^{14}$ Further evidence for this assignment can be derived from the dependence of the amplitude connected with the 7.4 ns component on the number of chromophores and the dependence on the emission wavelength $\left(\lambda_{\mathrm{flu}}\right)$, respectively. As reported in Table 1 , this amplitude is $0.8 \%$ for G1R2 and increases to $4 \%$ for G1R4 at $\lambda_{\text {flu }}=600$ $\mathrm{nm}$. This is reasonable as the probability of formation of the "excimer-like" entity increases as the number of chromophores increases in the dendrimer. By detection at $\lambda_{\text {flu }}=725 \mathrm{~nm}$, the values of the amplitude for the $7.4 \mathrm{~ns}$ component increase to $1.3 \%$ for G1R2 and to $6.3 \%$ for G1R4. The larger contribution of that component at longer emission wavelengths is also consistent with a red-shifted fluorescence from excimer-like entities.

Size of the Dendrimers. Fluorescence depolarization measurements of the monochromophoric G1R1 give a singleexponential anisotropy decay time $\left(\Theta_{1}\right)$ of about 1 ns (Table 2) and can therefore be associated with the time constant of rotation $\left(\Theta_{\text {rot }}\right)$ of the whole molecule. The corresponding long polarization time constant $\left(\Theta_{1}\right)$ increases to $1.3 \mathrm{~ns}$ in G1R4. Thus, we can calculate the diameter of the spherical rotor $\left(d_{\text {rot }}\right)$

$$
d_{\text {rot }}=\sqrt[3]{\frac{6 V}{\pi}}
$$

using the Stokes-Einstein-Debye relation

$$
\Theta_{\mathrm{rot}}=\frac{V \eta}{\kappa T}
$$

where $\Theta_{\text {rot }}$ is the rotational relaxation time obtained from $\Theta_{1}$ according to eqs 8 and $9, V$ the hydrodynamic volume, $\eta$ the solvent viscosity, $\kappa$ the Boltzmann constant, and $T$ the temperature. ${ }^{23}$ On the basis of the usually applied assumption of a spherical volume, $d_{\text {rot }}$ can be calculated from eq 10 . Inserting the determined $\Theta_{\text {rot }}$ values of 1 to $1.3 \mathrm{~ns}$ from Table 2 in the above equation yields rotor diameters in the range of $\sim 24-26$ $\AA$ which are close to the values $(22-24 \AA)$ deduced from different conformations of G1R4 by Molecular Modeling ${ }^{30}$ assuming that the distance between the center of the chromophores and the $\mathrm{sp}^{3}$ core carbon determines the length of a dendrimer branch. The increase in $\Theta_{\text {rot }}$ or $d_{\text {rot }}$ reflects well the expected increase of the hydrodynamic volume with the introduction of more chromophores in the dendritic structure.

Intramolecular Excitation Energy Hopping. Energy transfer processes can be revealed by time-resolved anisotropy data. The large value for the limiting anisotropy $\left(r_{0}\right)$ of G1R1 confirms the parallel orientation of the absorption and emission transition dipole moment for a single chromophore.

In contrast to the fluorescence anisotropy decay of G1R1, which contains only one peryleneimide chromophore, a 100200 ps anisotropy decay component is observed in the multichromophoric dendrimers. Therefore, this fast depolarization process can unambiguously be related to excitation energy hopping among the identical chromophores. The time scale of $100 \mathrm{ps}$ for these processes suggests that the observed energy hopping occurs in terms of fluorescence resonance energy 
transfer (FRET). Within the framework of the Förster formulation $^{29,31-33}$ an effective interaction radius $\left(R_{0}\right)$ can be calculated from the steady-state spectra and the fluorescence quantum yield of the donor chromophore $\left(\phi_{\mathrm{D}}\right)$ with the following equations:

$$
R_{0}^{6}=8.875 \times 10^{-5} \frac{\Gamma^{2} \phi_{\mathrm{D}} J}{n^{4}}
$$

Here, $\Gamma^{2}$ equals $2 / 3$ for the assumed random orientation of the chromophores, $\phi_{\mathrm{D}}$ is the donor fluorescence quantum yield, $n$ is the refractive index of the solvent (1.496 for toluene), and $J$ is the spectral overlap integral defined by

$$
J=\frac{\int F_{\mathrm{D}}(\lambda)\left[\epsilon_{\mathrm{A}}(\lambda)\right] \lambda^{4} \mathrm{~d} \lambda}{\int F_{\mathrm{D}}(\lambda) \mathrm{d} \lambda}
$$

where $\epsilon_{\mathrm{A}}(\lambda)$ represents the molar extinction coefficient of the acceptor and $F_{\mathrm{D}}(\lambda)$ denotes the donor fluorescence spectrum on a wavelength $(\lambda)$ scale. The calculated values of $R_{0}=38 \AA$ and $J=2.5 \times 10^{14} \mathrm{M}^{-1} \mathrm{~cm}^{-1} \mathrm{~nm}^{4}$ using the spectral data for the monochromophoric G1R1 model compound are on the typical order of magnitude where FRET has been reported in numerous publications. ${ }^{34}$

Information about the rate constant of FRET $\left(k_{\mathrm{FRET}}\right)$ can be derived from the fast anisotropy decay time $\left(\Theta_{2}\right)$. However, to quantify $k_{\text {FRET }}$ we have to take into account the possibility of multiple energy transfer channels in the case of a multichromophoric system containing equal chromophores among which efficient dipole-dipole interaction occurs. According to the energy hopping model described by eqs $3-8$, the measured decay time $\Theta_{2}$ can be related to $k_{\text {eet }}=k_{\text {FRET }}$ by combining eqs 8 and 9 to $\Theta_{\text {rot }}=\Theta_{1}$ and eq 14, where the value of $i$ represents the number of chromophores fully interacting in both forward and backward direction.

$$
k_{\mathrm{FRET}}=\frac{1}{i \Theta_{2}}-\frac{1}{i \Theta_{1}}
$$

If we take G1R2 as the model system for energy hopping between two peryleneimide chromophores and further assume that the energy transfer occurs in both directions, then the rate constant $\left(k_{\mathrm{FRET}}\right)$ calculated from eq 14 with $i=2$ results in $k_{\mathrm{FRET}}=2 \mathrm{~ns}^{-1}$. Using this value for $k_{\mathrm{FRET}}$ we can calculate the expected anisotropy decay time $\left(\Theta_{2}\right)$ for the case of equally distributed and interacting chromophores in $\mathbf{G 1 R 3}(i=3)$ and G1R4 $(i=4)$, which gives 143 and $112 \mathrm{ps,} \mathrm{respectively.} \mathrm{These}$ results are in good agreement with the experimentally observed $\Theta_{2}=130$ ps of G1R3 and $\Theta_{2}=110$ ps of G1R4, which indicates the validity of the proposed model of energy hopping among all chromophores. The theoretical energy hopping model can further be tested by the FRET anisotropy contribution $\beta_{2} /$ $r_{0}$ (Table 2), which according to eq 8 should be equal to $(i-$ $1) / i$. While the values of the parameter $\beta_{2} / r_{0}$ for G1R2 (48\%) and G1R3 (63\%) agree quite well with the theoretically expected values $50 \%$ and $66 \%$, respectively, $\beta_{2} / r_{0}$ for G1R4 $(66 \%)$ is somewhat smaller than the theoretical value of $75 \%$. This might indicate that all the four chromophores in G1R4 are not interacting equally; i.e., the fourth chromophore does not contribute in the energy-hopping process with the same efficiency as the other three. On the other hand, the comparatively small value of $\beta_{2} / r_{0}$ for G1R4 can be due to the presence of the preformed dimer decaying with $7.4 \mathrm{~ns}$ (Table 1) and acting as a energy trap thus affecting the anisotropy decay.
On the basis of the same approximation of energy hopping in terms of FRET in the different dendrimers, i.e., full crosstalk among all chromophores in G1R2 and G1R3 but only cross-talk among mainly three chromophores in G1R4, the donor-acceptor distances $\left(d_{\text {FRET }}\right)$ as calculated with the Förster eq $15^{29,31-33}$ using eqs $12-14$ and the fluorescence decay time of the donor chromophore $\left(\tau_{\mathrm{D}}\right)$ are listed in Table 2. All calculated values of $d_{\text {FRET }}$ for the peryleneimide dendrimers are in the order of $26 \AA$ which is in good agreement with the average distance between two chromophores in different conformations obtained from molecular modeling results. This agreement further substantiates the validity of the above proposed model for energy-hopping mechanism in the present dendrimers.

$$
d_{\mathrm{FRET}}{ }^{6}=\frac{R_{0}{ }^{6}}{k_{\mathrm{FRET}} \tau_{\mathrm{D}}}
$$

However, with an increasing number of chromophores $r_{0}$ decreases from 0.38 in G1R1 to 0.31 in G1R2 and to 0.24 in G1R4. This means that an additional fast depolarization processes on a time scale below the time resolution of $10 \mathrm{ps}$ for the time-correlated single photon counting anisotropy experiments takes place. Most likely this is energy transfer between neighboring chromophores, which, due to their conformational freedom, can become very close lying in the order of $10 \AA$. In view of the determined overlap integral (eq 18) and the corresponding Förster radius (eq 12) the rate constant of FRET at such short distances is in the range of $1 \mathrm{ps}^{-1}$. Alternatively, the loss of limiting anisotropy may be related to direct excitation into a locally excited state of a preformed dimer which leads rapidly into a delocalized excimer-like state as has been discussed previously. ${ }^{15}$

\section{Conclusions}

The comparative steady-state and time-resolved study of a series of dendrimers with a rigid tetrahedral core containing a different number of chromophores at the rim reveals different intramolecular interactions between the chromophores. The fluorescence decays show emission from an "excimer-like" species with a time constant of $7.4 \mathrm{~ns}$, beside the emission of the individual phenyl-substituted perylenemonoimide chromophores with $4.25 \mathrm{~ns}$. A decrease in the limiting anisotropy for the dendrimers containing multiple peryleneimide chromophores indicates the presence of ultrafast energy transfer processes in these systems. The obtained rotational relaxation times varying between 0.95 and $1.3 \mathrm{~ns}$ are consistent with the size of the different molecules. Furthermore, an additional fast depolarization component with time constants in the range of 100 to 200 ps can be attributed to Förster resonance energy transfer among the attached chromophores, since it is observed only for the multichromophoric systems. This excitation energy hopping between the peryleneimide chromophores in II-IV can be understood by the calculated large value for the spectral overlap integral $\left(J=2.5 \times 10^{14} \mathrm{M}^{-1} \mathrm{~cm}^{-1} \mathrm{~nm}^{4}\right)$ associated with a sizable Förster radius of $3.8 \mathrm{~nm}$. From the anisotropy analysis, it is also shown that while there is forward and backward energy transfer among all chromophores in the bichromophoric G1R2 and the trichromophoric G1R3, the additional fourth chromophore in G1R4 seems to interact to a little lesser extent with the others.

Acknowledgment. The authors gratefully acknowledge the FWO and DWTC (Belgium) through IUAP-IV-11 and the European Science Foundation through SMARTON for their 
continuing support. S.M. and J.H. thank KUL and FWO, respectively, for postdoctoral fellowships. M.M. thanks the EC through TMR-Sisitomas for a postdoctoral fellowship.

\section{References and Notes}

(1) Newkome, G. R.; Moorefield, C. N.; Vögtle, F. Dendritic Molecules: Concepts, Synthesis, Perspectives; VCH: Weinheim, Germany, 1996.

(2) Matthews, O. A.; Shipway, A. N.; Stoddart, J. F. Prog. Polym. Sci. 1998, 23, 1 .

(3) Jansen, J. F. G. A.; De Brabander-Vanden Berg, E. M. M.; Meijer E. W. Science 1994, 266, 1226.

(4) Atwood, J. L.; Davies, J. E. C.; Macnicol, D. D.; Vögtle, F.; Lehn, J.-M. Comprehensive Supramolecular Chemistry; Pergamon Press: Oxford, U.K., 1996.

(5) Stinson, S. C. Chem. Eng. News 1997, 75, 28.

(6) Roberts, J. C.; Bhalgat, M. K.; Zera, T. R. J. Biomed. Mater. Res. 1996, 30, 53.

(7) Twyman, L. J.; Beezer, A. E.; Esfand, R.; Hardy, M. J.; Mitchell, J. C. Tetrahedron Lett. 1999, 40, 1743.

(8) Yeow, E. K. L.; Ghiggino, K. P.; Reek, J. N. H.; Crossley, M. J.; Bosman, A. W.; Schenning, A. P. H. J.; Meijer, E. W. J. Phys. Chem. B 2000, 104, 2596.

(9) Balzani, V.; Scandola, F. Supramolecular Photochemistry; Horwood: New York, 1990.

(10) Gilat, S. L.; Adronov, A.; J. Fréchet, J. M. Angew. Chem., Int. Ed. Engl. 1999, 38, 1422.

(11) Bar-Haim, A.; Klafter, J.; Kopelmam, R. J. Am. Chem. Soc. 1997, $119,6197$.

(12) Hofkens, J.; Maus, M.; Gensch, T.; Vosch, T.; Cotlet, M.; Köhn, F.; Herrmann, A.; Müllen, K.; De Schryver, F. C. J. Am. Chem. Soc. 2000, 122,9728 .

(13) Morgenroth, F.; Müllen, K. Tetrahedron 1997, 53, 15349-15366. Morgenroth, F.; Berresheim, A. J.; Wagner, M.; Müllen, K. Chem. Commun 1998, 1139-1140. Morgenroth, F.; Kubel, C.; Muller, M.; Wiesler, U. M.; Berresheim, A. J.; Wagner, M.; Müllen, K. Carbon 1998, 36, 833-837.

(14) Hofkens, J.; Latterini, L.; De Belder, G.; Gensch, T.; Maus, M.; Vosch, T.; Karni, Y.; Schweitzer, G.; De Schryver, F. C.; Herrmann, A.; Müllen, K. Chem. Phys. Lett. 1999, 304, 1.

(15) Karni, Y.; Jordens, S.; De Belder, G.; Schweitzer, G.; Hofkens, J.; Gensch, T.; Maus, M.; De Schryver, F. C.; Herrmann, A.; Müllen, K. Chem. Phys. Lett. 1999, 310, 73.
(16) Karni, Y.; Jordens, S.; De Belder, G.; Hofkens, J.; Schweitzer, G.; De Schryver, F. C.; Herrmann, A.; Müllen, K. J. Phys. Chem. B 1999, 103,9378

(17) Gensch, T.; Hofkens, J.; Hermann, A.; Tsuda, K.; Verheijen, W.; Vosch, T.; Christ, T.; Basché, T.; Müllen, K.; De Schryver, F. C. Angew. Chem., Int. Ed. Engl. 1999, 38, 3752.

(18) Zhang, H.; Grim, P. C. M.; Foubert, P.; Vosch, T.; Vanoppen, P.; Wiesler, U.-M.; Berresheim, A. J.; Müllen, K.; De Schryver, F. C. Langmuir 2000, 16, 9009 .

(19) Weil, T.; Wiesler, U. M.; Herrmann, A.; Müllen, K. J. Am. Chem. Soc. 2000, submitted for publication.

(20) Eaton, D. F. Handbook of Organic Photochemistry; Scaiano, J. C. Ed.; CRC Press: Boca Raton, FL, 1989; Vol. 1, p 231.

(21) Maus, M.; Rousseau, E.; Cotlet, M.; Schweitzer, G.; Hofkens, J. Van der Auweraer, M.; De Schryver, F. C.; Krueger, A. Rev. Sci. Instrum. 2001, 72,36 .

(22) Homemade program developed in a cooperation between The Management of Technology Institute (Belarusian State University) and The Division of Photochemistry and Spectroscopy (Katholieke University of Leuven).

(23) O'Connor, D. V.; Phillips, D. Time-Correlated Single Photon Counting, Academic Press: London, 1984; p 252.

(24) Jablonski, A. Acta Phys. Pol. 1970, A38, 453

(25) Berberan-Santos, M. N.; Valeur, B. J. Chem. Phys. 1991, 95, 8048.

(26) Berberan-Santos, M. N.; Canceill, J. Gratton, E.; Jullien, L.; Lehn,

J.-M.; So, P.; Sutin, J.; Valeur, B. J. Phys. Chem. 1996, 100, 15.

(27) Berberan-Santos, M. N.; Choppinet, P.; Fedorov, A.; Jullien, L.; Valeur, B. J. Phys. Chem. 1999, 121, 2526.

(28) Brodard, P.; Matzinger, S.; Vauthey, E.; Mongin, O.; Papamicaël, C.; Gossauer, A. J. Phys. Chem. A 1999, 103, 5858.

(29) Resonance Energy Transfer; Andrews, D. L., Demidov, A. D., Eds.; John Wiley \& Sons: Chichester, U.K., 1999.

(30) Spartan Program, Wave function, Inc., 17401 Von Karman Ave., Ste. 370, CA 92612.

(31) Förster, T. Ann. Phys. (Leipzig) 1948, 2, 55.

(32) Duus, J. Ø.; Meldal, M.; Winkler, J. R. J. Phys. Chem. B 1998, $102,6413$.

(33) Cheung, H. C. Topics in Fluorescence Spectroscopy; Lakowicz, J. R., Ed.; Plenum Press: New York, 1991; Vol. 2, p 127.

(34) A collection of various references can be found in the following: Haughland, R. P. Handbook of Fluorescent Probes and Research Chemicals, 6th ed.; Molecular Probes Inc.: Eugene, OR, 1996; p 46. 